\title{
La gramática de la primera lengua en la escuela: Reflexiones sobre su enseñanza-aprendizaje y sobre el contenido gramatical escolar
}

\author{
Xavier Fontich Vicens, Universitat Autónoma de Barcelona, España
}

Artículo recibido el 21 de julio de 2013, versión final recibida el 30 de agosto 2013

\begin{abstract}
En este artículo presentamos algunas reflexiones sobre la enseñanza de la gramática en primera lengua en la escuela obligatoria. Nos situamos entre los estudios que defienden que la instrucción gramatical ayuda a los alumnos a mejorar su capacidad de reflexionar sobre la lengua y de usarla. Exponemos tres aspectos que la investigación puede abordar para conocer mejor qué caminos pueden llevarnos a desarrollar en el aula una instrucción gramatical fructífera y educativa: (a) el aprendizaje de la gramática, (b) el contenido de enseñanza y aprendizaje gramatical, y (c) los procesos de enseñanza.
\end{abstract}

\section{Primeras ideas: Saber lengua, saber gramática ${ }^{1}$}

Plantearse el rol que la gramática juega en el aprendizaje de la lengua nos sitúa ante de preguntas previas como “¿Qué quiere decir saber lengua?”, “¿Saber lengua significa saber usar la lengua?”, “Significa tener conocimientos sobre el sistema?”, “¿Saber lengua es saber gramática o es también saber gramática?”. Algunas investigaciones sugieren dos ideas complementarias: por una parte, que el conocimiento sobre el sistema que se desprende de la lengua en uso no es un conocimiento sistematizado y no permite al aprendiz recuperar este saber en situaciones de uso formal; y por otra parte que el conocimiento del sistema gramatical no garantizará tampoco saber usar la lengua (Ribas, Milian, Guasch y Camps, 2002; Camps, 1998; Trévise, 2010; Camps, 2010a). Esos estudios parten de la idea según la cual la gramática es importante y forma parte del conocimiento relativo al uso de la lengua. Entre el uso y el conocimiento del sistema existen vínculos de naturaleza diversa y es crucial que la enseñanza muestre a los aprendices caminos para relacionar las dos caras de la misma moneda (Camps, Guasch, Milian y Ribas, 2005).

C. Rodríguez y M. Milian explican esta relación en términos metafóricos. Rodríguez (2000) considera que podemos conducir sin tener conocimientos del motor de un vehículo igual que podemos hablar sin conocimiento explícito de la gramática explícita, aunque para el hablante sí será necesario conocer el sistema en caso de tener que usar la lengua en situaciones formales (como la escritura de textos técnicos). Asimismo, Milian ${ }^{2}$ compara el uso que hacemos de una silla y de la lengua: una silla 
sirve para sentarse en ella (uso), pero si nos dedicamos a vender sillas tendremos que atender a aspectos como los materiales de los que se compone según los usos, de los instrumentos con que los podemos elaborar sillas, del diseño y la ergonomía o de los colores de moda del momento (sistema); también nuestro conocimiento de la lengua va a centrarse necesariamente tanto en los aspectos de uso como en los aspectos gramaticales o de sistema. Ambas metáforas apuntan al hecho según el cual el conocimiento explícito de la lengua nos será imprescindible en aquellas situaciones comunicativas altamente formales (un científico que tiene que escribir un resumen o un locutor radiofónico que tiene que atender cuestiones de dicción y de normativa), que convivirán con situaciones informales en las que el foco consciente sobre el sistema no será necesario (el científico y el locutor se encuentran para tomar un café o para rellenar un formulario).

Una parte de la investigación sobre enseñanza gramatical ha remarcado cuatro ámbitos en los que la instrucción de la gramática es imprescindible (cf. Fontich, 2006, para una síntesis): la composición escrita y la expresión oral formal en primeras lenguas; la adquisición de nuevas lenguas y el contraste interlingüístico con aquellas que ya conocemos; la ortografía y aquellos aspectos normativos ligados a la oración y a la relación de las palabras en el texto; y finalmente la cultura general que tenemos que esperar de cualquier ciudadano escolarizado y de profesionales no directamente relacionados con la lengua que deberán manejar diccionarios, traductores o libros de estilo. Sin embargo, por lo que respecta a la relación entre gramática y composición escrita estudios como los citados en Andrews et al., (2004a y 2004b) niegan o minimizan la incidencia de la instrucción gramatical en la mejora del uso escrito de los alumnos y proponen únicamente la combinación de oraciones (sentence combining) como medio realmente eficaz para lograr textos más complejos. Mientras que Weaver (2010) o van Gelderen (2010) se sitúan en esta posición y defienden una instrucción implícita de la gramática orientada al uso, trabajos como los de Janks (2010), Freeman y Freeman (1998) o Myhill (2011) desde posiciones distintas consideran imprescindible la reflexión explícita sobre la gramática y la capacidad de los escolares para organizar un sistema coherente.

Janks (2010) se sirve de la lingüística sistémico-funcional para mostrar la necesidad de ver las formas gramaticales como recursos para vehicular los significados y las intenciones de los hablantes. La autora muestra cómo este planteamiento puede 
traducirse en prácticas en el aula centradas en el estudio oracional sin caer en rutinas de simple etiquetaje e identificación, promoviendo además en clase la conciencia crítica y el debate sobre aspectos ideológicos del uso de las lenguas. De una manera similar y desde la perspectiva de la gramática tradicional, Freeman y Freeman (1998) proponen actividades también centradas en la oración y en las que el aprendiz tiene un rol activo determinante, puesto que debe recoger datos gramaticales diversos, sistematizarlos y llegar a conclusiones que darán cuenta de la coherencia del sistema.

La posición de Myhill (2011) es distinta, por cuanto esta autora explora una gramática del uso para ayudar a los alumnos a reflexionar sobre los problemas que surgen a lo largo del proceso de escritura. Sin embargo comparte con las autoras precedentes su visión sobre la importancia de la reflexión metalingüística. Su estudio se centra en la escritura de tres géneros discursivos, para cada uno de los cuales se propone explorar unos aspectos gramaticales concretos: la primera y la tercera persona para trabajar el punto de vista en la narración, los conectores y la subordinación en la argumentación, y la selección léxica en el poema. Las mejoras en los textos resultantes al cabo de tres semanas muestran que la instrucción gramatical puede ser beneficiosa para mejorar las destrezas escritas de los alumnos.

Estos últimos tres estudios representan orientaciones interesantes para ampliar el debate sobre la instrucción gramatical. De ellos se desprenden tres ideas. Primera: la adopción de los planteamientos de la linguiística funcional permite definir mejor qué contenido gramatical para la escuela, más allá de una gramática descontextualizada y formal inspirada en mayor o menor grado en los modelos estructuralistas y generativistas. Segunda: un cambio metodológico en el aula apunta a escenarios con más participación de los alumnos para estimular la reflexión, con atención al tipo de interacción oral desarrollada en pequeño grupo. Tercera: la exploración explícita de microsistemas gramaticales (conectores, tiempos verbales, etc.) puede guiar la reflexión gramatical del alumno mientras escribe, es decir debemos poner la selección de conceptos gramaticales en relación a los objetivos comunicativos. En relación a este último punto, podemos concluir que coexisten múltiples aproximaciones a la complejidad gramatical, algunas más ligadas al uso y otras más ligadas al sistema, pero todas ellas susceptibles de colaborar a construir el conocimiento metalingüístico de los alumnos (Camps et al., 2005). 
Todo ello nos lleva a adoptar una perspectiva acorde con los postulados ecológicos y semióticos del aprendizaje linguiístico (van Lier, 2004) según los cuales el desarrollo lingüístico no puede desligarse del desarrollo humano en contextos de interacción ricos. Para el aprendizaje de lenguas (primeras o segundas) esto implica ir más allá de la instrucción directa y crear contextos ricos con múltiples 'oportunidades' (affordances) (van Lier, 2008) para que puedan intervenir alumnos con diversas capacidades y en los que la reflexión gramatical también se dé en escenarios diversos. Implica una concepción de la gramática no únicamente formal, sino integrada por los aspectos morfosintácticas, semánticos y pragmáticos (van Lier, 1995; Larsen-Freeman, 2003).

\section{Aprender gramática en la escuela}

En España, la relación entre gramática y uso ha sido explorada de forma especial por el grupo GREAL ${ }^{3}$, del Departamento de Didáctica de la Lengua i la Literatura de la Universitat Autònoma de Barcelona, España. Este grupo empezó analizando en los años noventa los procesos de composición escrita de los alumnos trabajando en pequeño grupo a partir del modelo de Secuencia Didáctica (SD, Camps, 1994). Estos estudios sobre la composición escrita pusieron de relieve aspectos interesantes ligados al uso y al aprendizaje de la escritura: la incidencia de los contextos de producción en el proceso de escritura, la evaluación formativa, la noción de 'texto intentado' o la escritura en segundas lenguas (Milian, 2004; Guasch, 2001). Pusieron de relieve también los problemas de los escolares a la hora de manejar los conceptos gramaticales en la revisión del escrito. Esto supuso introducir en el programa de investigación del grupo el estudio de la actividad metalingüística de los escolares y la construcción de los conceptos gramaticales.

A lo largo de la última década el grupo ha desarrollado varios estudios centrados en la construcción del saber gramatical de los alumnos, combinando metodologías y focos de interés diversos: entrevistas semi-estructuradas a los alumnos fuera del aula a partir de pequeñas actividades (Camps, 2000; Notario, 2001), estudios longitudinales (Gonzalvo y Camps, 2003) y estudios centrados en el diálogo en situaciones naturales de aula (Ribas et al., 2002; Milian y Camps, 2006; Fontich, 2011), en el seno del modelo de Secuencia Didáctica (SD) (ver más abajo para una descripción sumaria de este modelo). 
Los resultados coinciden con estudios llevados a cabo en el contexto anglosajón (Myhill, 2000) y francófono (Kilcher-Hagedorn, Othenin-Girard, de Weck, 1987; Martin, 1999; Fisher, 1996, 2004). Señalan que el saber gramatical de los escolares es muy heterogéneo y diverso, que no se trata de una cuestión de todo o nada sino que es diverso y poco coherente, que no siempre puede ser justificado, y que a menudo es fruto de la fosilización de prácticas escolares o de la pobreza de los libros de texto. Señalan asimismo la dificultad de muchos alumnos para separar lengua y realidad, la visión lineal de las relaciones entre las palabras, y la separación entre el conocimiento declarativo y el procedimental.

Mientras que las investigaciones citadas por Andrews (2010) y sus colaboradores (Andrews et al., 2004a, 2004b) que hemos mencionado niegan o minimizan la incidencia de la instrucción gramatical en la mejora del uso, los estudios sobre los conceptos gramaticales van más allá, pues ponen de relieve que esta instrucción ni tan siquiera sirve para construir un saber gramatical coherente. Mientras que aquellos autores proponen dejar de lado toda instrucción gramatical, estos últimos presentan sus estudios como el punto de partida para buscar qué caminos pueden llevarnos a una mejor conexión entre instrucción de la gramática, construcción del saber gramatical y uso de la lengua, abriendo así una importante nueva avenida para la investigación. Remarcan que la escuela debe tener en cuenta la relación estrecha pero no directa entre enseñanza gramatical y uso de la lengua, y que es básico que los alumnos hablen de los fenómenos gramaticales para aprender gramática.

Efectivamente, una de las observaciones colaterales realizadas por los estudios sobre los conceptos gramaticales es que la conversación con el adulto-investigador facilita que el alumno verbalice su reflexión metalingüística, lo que sugiere que en el marco escolar el diálogo debe ser uno de los pilares del aprendizaje. Esto apuntaría a la necesidad de promover escenarios de aula en los que se hable para aprender gramática, combinando niveles diversos de explicitación (Nadeau y Fisher, 2011).

Los estudios centrados en el diálogo en el aula destacan las dificultades para desarrollar escenarios verdaderamente dialógicos en los que el habla del docente sea algo más que un discurso transmisivo. Edwards y Mercer (1987) muestran cuán difícil es que este discurso se oriente a lo que ellos llaman "conocimiento de principios" (generalizable y autónomo) más allá del "conocimiento ritual" (ligado al contexto de aprendizaje). En esta misma línea, otros estudios muestran la dificultad que tienen los 
alumnos para desarrollar nociones abstractas, a pesar de los esfuerzos de los docentes para actuar de forma contingente y llevar a los escolares a la construcción de los conceptos científicos más allá de las nociones espontáneas (en términos vygotskyanos). Asimismo, la investigación sobre el diálogo entre alumnos trabajando en pequeños grupos muestra el potencial que los escenarios colaborativos tienen para aprender gramática.

Varios autores han indicado la necesidad de repensar la noción vygotskyana de "zona de desarrollo próximo" cuando nos situamos en el contexto educativo formal (Wells, 2001). En este contexto los procesos de enseñanza y aprendizaje se dan por lo general en grupos numerosos, con una gran heterogeneidad de historias personales, y en los que el discurso del profesor es preponderante. Desarrollar procesos de andamiaje en los que progresivamente se ceda la palabra a los alumnos significará crear contextos ricos en los que haya múltiples "oportunidades" (affordances) de participación para aprendices con capacidades variadas y en las que cada alumno adopte distintos roles: 'ayudar al que sabe menos', 'ayudar a mi igual', 'dejarme ayudar por el que sabe más' y 'movilizar lo que sé' (experiencias, datos memorizados, analogías, etc.) (van Lier, 2004). A través de esta dinámica los alumnos se integran en un contexto de trabajo y contribuyen a crear este contexto compartido, entendido como una zona de desarrollo intermental (Mercer y Littleton, 2007). Para ello será imprescindible el trabajo con las reglas básicas del habla exploratoria (ground rules) para que los alumnos superen las situaciones en las que la interacción no permite el aprendizaje (Mercer y Littleton, 2007). Los siguientes estudios de Méndez (2011), Milian y Camps (2006) y Fontich (2011 y 2013) exploran aspectos diversos del habla en el aula.

Méndez (2011) explora el diálogo sobre la oración que una docente mantiene con alumnos de primaria. A pesar de la intensa actividad de los niños y de la satisfacción con la que éstos valoran la sesión (coincidiendo con otros docentes observadores) un análisis atento de la interacción revela que el esquema subyacente ha sido predominantemente el de IRF (Initiation-Response-Follow-up, Sinclair y Coulthard, 1975), en un contexto de preguntas cerradas que los alumnos concebían como preguntas adivinanza y que impedían a la maestra (condicionada por un guión preestablecido) recoger las interesantes aportaciones y dudas expresadas por los niños y niñas. 
Por su parte, Milian y Camps (2006) exploran los diálogos en gran grupo en el seno de una secuencia didáctica de gramática que unos docentes mantienen con sus alumnos en relación a aspectos diversos (los conectores, el tiempo de la narración, etc.). En relación con la noción de tiempo de la narración, la profesora se sitúa en niveles de abstracción diversos, alto ("palabras que indiquen temporalidad") y bajo ("palabras que den idea de cuándo se producen los hechos"), en un doble movimiento no lineal sino recursivo y contingente con las respuestas de los alumnos. A pesar de la intensa interacción y participación los alumnos no superan niveles bajos de abstracción.

Por último, también en el seno de una secuencia didáctica, Fontich (2013) muestra que la interacción oral en pequeño grupo es un escenario óptimo para el aprendizaje y que los alumnos son capaces de desarrollar tramas argumentativas ricas si trabajan en colaboración y sobre la base de las reglas básicas del habla exploratoria (Mercer y Littleton, 2007). En Fontich (2011) se exploran unos diálogos desarrollados en otra secuencia didáctica y se desarrolla un modelo de análisis de la interacción a partir de la siguiente idea: una interacción rica en el plano argumentativo es lo que va a permitir que los alumnos integren en la interacción conceptos metalingüísticos necesarios para solucionar el problema. Se pone así sobre la mesa la necesidad de desarrollar instrumentos para describir cómo el conocimiento se va transformando a lo largo de la interacción oral en pequeño grupo y en situación natural de clase (Kumpulainen y Mutanen, 2000; Schwarz, Dreyfus, y Hershkowitz, 2009), pues aunque quizás no se llegue a la solución los intentos de integración de nociones gramaticales pueden concebirse como aprendizaje en grados diversos. Para ello, como ya hemos mencionado debemos tomar consciencia de que no es suficiente disponer a los alumnos en grupo para que esta argumentación se desarrolle de forma constructiva (Mercer, 2008), por lo que deberemos acompañar a los alumnos para que sepan cómo desarrollar una interacción verdaderamente colaborativa (Edwards y Mercer, 1987; Wegerif, Boero, Andriessen y Forman, 2009).

\section{Qué gramática para la escuela}

Uno de los interrogantes recurrentes planteados en relación a la enseñanza y aprendizaje de la gramática es si debemos tomar como modelo de referencia los estudios lingüísticos o si una gramática para la escuela debería desarrollar su propio modelo. En el contexto español para la enseñanza de catalán, las dos obras básicas que nos sitúan en 
esta tesitura son Desclot (1977) y Camps (1986). Las dos obras abordan el trabajo sobre nociones oracionales básicas.

Desclot (1977) parte de los postulados lingüísticos percibidos como hegemónicos (los modelos generativistas del momento) para adaptarlos en bloque al contexto escolar, en una línea similar a las propuestas de Dubois (1969) para la enseñanza del francés. Por el contrario, el trabajo de Camps (1986) parte de este contexto escolar para plantear caminos que ayuden a dar respuesta a las necesidades de aprendizaje. Es esta segunda perspectiva la que encontramos ampliamente desarrollada y validada por la investigación posterior en estrechada colaboración con docentes de distintos niveles (Camps y Zayas, 2006; Bronckart, 2008; Vargas, 2009). Sin embargo ha sido el planteamiento representado por Desclot (1977) el que está plenamente instalado en libros de texto, en el currículo e incluso en el imaginario colectivo de los docentes, como ha sucedido en otros contextos (Andrews, 2010; French, 2010).

Debemos continuar señalando, por lo tanto, que la escuela y la lingüística tienen objetivos diferentes. Los objetivos de una gramática escolar y de las teorías científicas no son ni pueden ser coincidentes. La teoría es un modelo de la realidad que no es la realidad. La escuela tendrá que recurrir a la teoría no en bloque sino eligiendo aquellas nociones más rentables para sus objetivos, el más básico de los cuales es la formación lingüística de los alumnos y el desarrollo de un repertorio amplio de usos comunicativos (Camps, 1998; Zayas, 2006; Bronckart y Schneuwly, 1991; Locke, 2009, 2010a y 2010b). Para lograr este objetivo el conocimiento gramatical es imprescindible, pero entendido como un conocimiento orientado no tanto al estudio del sistema gramatical per se como a la reflexión gramatical (Larsen-Freeman, 2003; Milian, 2005; Brucart, 2000). En este sentido, hay que dejar de pensar que serán los modelos lingüísticos los que nos indicarán cómo actuar en el aula. Hace falta redimensionar aspectos diversos: saber que no podemos capturar con una sola mirada la complejidad del sistema gramatical; identificar claramente los objetivos de estudio de los aspectos parciales que los alumnos tienen que conocer (la correlación temporal, la relación entre verbo y complementos, etc.); hacer participar a los alumnos en la recogida de datos que posteriormente serán observados y que nos permitirán contrastar, por ejemplo, el oral y el escrito; o mostrar que la oración no se reduce a un conjunto de relaciones morfosintácticas sino que también intervienen en ella aspectos semánticos y 
pragmáticos, es decir aspectos ligados al significado de las palabras y a las intenciones del hablante.

Podemos ejemplificar este último aspecto (la relación entre morfosintaxis, semántica y pragmática) como sigue. Observemos la siguiente oración: "Al final del partido llovieron todo tipo de objetos sobre el árbitro". Se trata de una oración muy real en español, con una presencia peculiar del verbo. En un uso prototípico este verbo tiene un sentido meteorológico y no admite complementos regidos (semántica), y es defectivo de plural y de primera y segunda persona (morfosintaxis); sin embargo la oración es del todo aceptable, un uso desviado si se quiere que responde a unas intenciones muy claras del hablante (pragmática). Estas intenciones no podrían satisfacerse con dos verbos cercanos como "caer" y "lanzar". Efectivamente, "caer" puede connotar un número elevado de elementos implicados pero también un número reducido, a diferencia de "llover": precisamente (en español peninsular) decimos "Caen cuatro gotas" y no "Llueven cuatro gotas". Por su parte, "lanzar" implica la presencia de un agente, aunque no lo explicitemos, mientras que "llover" nos permite esconderlo del todo.

¿Quién podría recurrir a una oración así? Sin duda un observador muy parcial que quiere minimizar el comportamiento incívico de los espectadores. El aclamado periodista y escritor barcelonés Manuel Vázquez Montalbán (1939-2003) lo hace en la memorable crónica del partido de fútbol de cuartos de final de copa entre F.C. Barcelona y R. Madrid de 1970, en el que el famoso y polémico árbitro Emilio Guruceta silba un penalti inexistente a favor del Real Madrid y a tres minutos del final el partido se suspende por invasión del campo: "La lluvia de almohadillas es impresionante (...) Hoy es fiesta, se respira libertad y la noche tiene los colores más propicios". El uso de "lluvia" responde a unas intenciones muy claras. Y en nuestra oración el uso de "llover" tiene las siguientes consecuencias en el plano morfosintáctico: concuerda con un sujeto en plural y pospuesto ("todo tipo de objetos"); exige la obligatoria presencia de la preposición "sobre" (sería extraño decir "Llueven bajo el árbitro") que nos permite reflexionar sobre el Complemento Preposicional; y exige en catalán sustituir el núcleo del SN por el pronombre partitivo 'en' propio del Complemento Directo indefinido ("En plovien de tot tipus, d'objectescomo pasa con los verbos inacusativos ("En queien de tot tipus, d'objectes").

Algunos autores señalan la necesidad de superar la visión estrictamente formal de la gramática oracional, heredera de la autonomía de la sintaxis de algunas propuestas 
generativistas. Proponen entender la gramática desde un punto de vista funcional como la unión orgánica de morfosintaxis, semántica y pragmática. Esto nos permitirá abordar los fenómenos en base a la observación y la discusión. Milian (2005) utiliza la expresión "hablar para hacer gramática" y Larsen-Freeman (2003) propone el neologismno "grammaring", considerando necesaria la integración orgánica de forma, semántica y pragmática. Van Lier (1995) propone un esquema que responde a la misma idea, según el cuál usar la lengua sería como pasar un decodificador sobre un código de barras: todos los elementos (los formales, los semánticos y los pragmáticos) interaccionan y participan en la construcción del sentido y la comunicación.

\section{Abordar la enseñanza de la gramática en el aula de primera lengua}

Estudios como los de Gil y Bigas (2009), Gil (2010; 2011) o French (2010) muestran cómo escolares de primer ciclo de primaria (7-8 años) son capaces de generar una reflexión gramatical rica, original e interesante. Una de las actividades propuestas por Gil y Bigas (2009) es un dictado en pareja en el que las palabras no están segmentadas. Comparan la reflexión desencadenada con la de niños y niñas de $4^{\circ}\left(9-10\right.$ años) y $6^{\circ}$ (11-12 años) en la misma actividad y remarcan que a medida que se sube de grado la reflexión metalingüística deviene más pobre y menos exploratoria. Los alumnos más pequeños muestran iniciativa y curiosidad y avanzan posibles explicaciones, pero esto se pierde cuando son mayores. Estos estudio nos sugieren interrogantes como "¿Por qué pasa esto?”, “¿Por qué cuesta tanto la reflexión gramatical?”, “¿Por qué en cursos superiores no se mantiene el grado de implicación que podemos encontrar en cursos iniciales?”, “QQue hacemos mal?”, “QQué podemos hacer para mejorar?”. Un trabajo como el de Rodríguez (2011), centrado en alumnos de secundaria, suscita interrogantes parecidos.

Una posible respuesta es la correlación entre el interés de los niños por la gramática y su interés por el mundo en general. Este mundo va siendo progresivamente más complejo y requiere de procedimientos que guíen nuestra mirada y faciliten el paso a una observación paulatinamente más sofisticada y estructurada, que permita mayores niveles de integración del saber. Esto pasaría en todas las materias escolares: también en la gramática. Aquí es donde fallaríamos. La escuela no encuentra maneras de articular esta curiosidad sobre la lengua y proporcionar ayudas que la estimulen. Afinando más por este camino, una segunda respuesta hace referencia a las razones por las que la 
escuela no encuentra las maneras de proporcionar ayudas efectivas para los alumnos. En las líneas precedentes hemos aludido a la necesidad de un mayor conocimiento sobre el saber gramatical de los alumnos, así como al modelo de gramática para la escuela. Otra posible razón hace referencia a la metodología de aula.

En el aula deben desarrollarse procedimientos adecuados para la actividad metalingüística, atenuando la instrucción directa a favor de la acción y la experiencia (Camps y Ferrer, 2000). Así, para aprender gramática el alumno elabora y utiliza instrumentos de evaluación, recoge datos, manipula, compara, sistematiza, discute, comparte, contrasta, expone, escribe... en definitiva "hace cosas", con la guía siempre del profesor. El habla del alumno y la escritura van a tener una gran importancia, por lo cual el dispositivo didáctico deberá identificar y trabajar en la medida de lo posible los rasgos de los géneros textuales implicados tanto orales (el habla exploratoria, la exposición formal) (Mercer y Littleton, 2007; Vilà, 2004) como escritos (el diario de aprendizaje, el informe final o el comentario de un gráfico) (Wells, 2001). Esto significa dar el protagonismo en el aula al alumno. Los puntos anteriores mejoran el nivel de implicación y motivación del alumno (Bergin, 1999), que ve que no es un simple terminal en un esquema transmisivo. Percibe que su rol ahora es el de protagonista y generador de conocimiento para si mismo y para los demás.

Por otro lado, se ha indicado la necesidad de que el docente gestione el aula para que sea un espacio de intercambio, sabiendo que este intercambio no se va a producir por el simple hecho de disponer físicamente en grupo a los alumnos (Mercer, 2008). Asimismo debe evitar maximalismos que lo puedan inhibir y adecuándose de forma realista a las circunstancias de partida (hábitos de trabajo, posibilidades de espacio, número de alumnos, puntos de acuerdo con otros profesores...) debe intentar crear las condiciones para que el alumno sepa qué se espera de él cuando está en grupo y para que aprenda a convertirse en protagonista que interviene, escucha, rebate y colabora. El docente sabe qué impartir (la parte correspondiente del currículo), cómo hacerlo (a partir de ideas sobre cómo presentar un determinado punto gramatical de una manera más eficiente) y por qué hacerlo así (ha identificado dificultades de comprensión). En este punto prepara una puesta en escena para aprender a través de la autorregulación y la cooperación con los demás, en un proceso de intercambio en el que el alumno a la vez que aprende también enseña a los demás (Rabatel, 2004). 
Trabajos como Camps y Zayas (2006) o Camps (2010b) subrayan la necesidad de plantear nuevas maneras de enseñar gramática como una hipótesis para su aprendizaje. ¿Una manera más constructivista que contemple un cambio metodológico permitiría mejorar el aprendizaje de la gramática? Es decir: la metodología como contenido también de enseñanza y aprendizaje y no como una simple opción cosmética. Como ya ha sido señalado, una propuesta de intervención en el aula para enseñar gramática tiene que poner a los alumnos en situación de comunicarse para aprender y a la vez constituir para el docente un instrumento de evaluación formativa, un espacio de reflexión y una herramienta para sistematizar el saber gramatical. El grupo GREAL ha trabajado sobre el modelo de la Secuencia Didáctica de Gramática (SDG).

Este modelo supera una visión puramente transmisiva de la enseñanza de lenguas y la consideración del papel activo y constructivo del aprendiz, remarcando la necesidad de aproximaciones globales a la enseñanza de la gramática más allá del ejercicio o la tarea aislada y puntual. Plantea el trabajo por proyectos también para el aprendizaje gramatical y la necesidad de promover situaciones de enseñanza orientadas a una interacción diversa y rica y a la participación de los aprendices en los procesos interactivos, adoptando una perspectiva sociocultural. Integra discurso oral y escrito así como uso y reflexión sobre el uso y busca también la diversidad en las oportunidades de aprendizaje y por lo tanto la posibilidad de participación de aprendices con habilidades diversas. Asimismo articula un conjunto de actividades que están orientadas siempre a un objetivo, que responde a la vez a dos preguntas: “¿Qué tiene que hacer el alumno?” y “¿Qué tiene que aprender?”. Organiza la secuencia de actividades en tres fases, de preparación, de ejecución y de cierre, con la elaboración de un informe final. Se rige por el criterio de elementalidad, según el cual un núcleo inicial de nociones operativas dará paso a un conjunto subsiguiente de nociones, progresivamente más complejas y articuladas a partir de aquel núcleo inicial. Y prevé finalmente tres submodelos de intervención según si el foco se sitúa en el uso, en el sistema o en el contraste entre lenguas o variantes de una misma lengua. Los tres submodelos parten de la idea según la cual diferentes aproximaciones a la gramática contribuyen a articular conocimientos gramaticales también diversos.

\section{El modelo de Secuencia Didáctica de Gramática: Enseñanza e investigación}


Este modelo ha sido contrastados ampliamente en el aula por profesores de distintos niveles y a continuación ponemos varios ejemplos de ello, a partir de las experiencias recogidas en Camps y Zayas (2006) y Manresa y Durán (2011).

(1) Foco situado en el uso: Los alumnos deben explorar un género discursivo concreto y para ello el profesor focaliza la atención en aspectos gramaticales complejos que deben ser dominados para conseguir un texto elaborado. Por ejemplo, en relación al artículo periodístico y al cuento, deberán contrastar las propiedades textuales y aspectos oracionales diversos. En relación al artículo periodístico, trabajarán los titulares a partir de patrones semánticos oracionales. O en relación a reseñas de libros deberán trabajar la cohesión, combinando actividades de transformación, de textualización y de integración de textos diversos.

(2) Foco situado en el contraste: Los alumnos exploran la dimensión dinámica de la lengua y para ello observan de qué manera se manifiesta el cambio, ya sea contrastando variantes dialectales de una misma lengua, ya sea contrastando lenguas entre sí para observar fenómenos de interferencia. También el contraste entre lenguas sirve para observar fenómenos concomitantes, como las funciones sintácticas. Los alumnos trabajan, por ejemplo, la variación dialectal de determinados pronominales, que pueden encontrarse en recesión. También razonan sobre el diferente comportamiento de dos lenguas en cuanto a determinados usos verbales. O contrastan los resultados de traductores automáticos.

(3) Foco situado en el sistema de la lengua: Los alumnos exploran conceptos oracionales básicos, así como nociones ligadas al texto. Abordan por ejemplo la oración desde una perspectiva léxica y relacionan una lista de oraciones con una lista de patrones semánticos. Clasifican los verbos a partir del número de complementos que reclaman. O discuten qué entienden por oración.

El modelo de Secuencia Didáctica de Gramática ha sido llevado a término en aulas diversas y ha constituido también en algunos casos el marco para desarrollar investigaciones en profundidad. Seguidamente detallamos la experiencia de Rodríguez (2011) sobre los tiempos del pasado en los textos narrativos en secundaria.

La profesora propone la escritura de una experiencia vivida ("Mi mejor verano") con el objetivo de que el alumnado a partir de un trabajo de revisión del texto amplíe su 
repertorio de tiempos verbales. Observa que los alumnos completan correctamente una actividad gramatical sobre identificación de un amplio abanico de tiempos del pasado (saber declarativo) pero que no son capaces de usar este conocimiento en la escritura de la narración (saber procedimental), por cuanto los alumnos se limitan a usar imperfecto (“cantaba") y pretérito perfecto (“canté"). Estudios como Camps (2000) o Fisher (2004) constatan también la separación entre el saber declarativo y el saber procedimental.

La investigación contrasta algunos diálogos recogidos en tres momentos del proceso de escritura: la planificación, la revisión del texto de un compañero, y la revisión del propio texto. Se observa que la mayoría de alumnos $(60 \%)$ no incorporan a la planificación del escrito ningún tiempo del pasado distinto del perfecto simple y del imperfecto, a pesar de las actividades gramaticales iniciales completadas con éxito. Por el contrario, en la revisión final del texto, realizada individualmente después de la revisión entre iguales, las cifras se invierten y es ahora el $61 \%$ de los alumnos el que consigue enriquecer su texto incorporando otros tiempos. Esto sugiere que la revisión gramatical entre iguales proporciona al alumno el contexto de aprendizaje adecuado para mejorar sus relatos.

Sin embargo la investigación revela una cuestión interesante. A pesar de la mejora tras la revisión individual final, los alumnos muestran muy poca capacidad de sugerir mejoras en la revisión intermedia de los textos de los compañeros. La investigadora interpreta esto como sigue. En la primera versión del relato el esfuerzo y la atención de los alumnos se centran básicamente en el control semántico-pragmático del texto ('qué digo, qué es conveniente decir'), sin poder atender al mismo tiempo a los aspectos gramaticales objeto de enseñanza, coincidiendo con lo observado ya por los primeros estudios sobre los procesos de composición escrita desarrollados en los años ochenta. La revisión entre iguales ha provocado un cambio de perspectiva necesario: el alumno que revisa abandona la posición de enunciador preocupado por el contenido para situarse en la de observador externo, que focaliza su mirada en los objetivos de aprendizaje gramatical. A pesar de ello no es capaz de sugerir mejoras en los textos revisados porque la introducción de cualquier cambio conlleva reorganizar el texto. Finalmente, cuando vuelve a asumir la posición de enunciador en su propio relato, lo hace consciente de los objetivos gramaticales y es su control del contenido lo que le permite incorporar más tiempos del pasado. 
Esta experiencia muestra cómo la revisión del texto ha propiciado un contexto rico en el que orientar el estudio de la gramática al uso lingüístico. Los resultados indican la importancia del diálogo y la reflexión en el aula, así como la necesidad de no separar la enseñanza de los procesos de investigación. El estudio se sitúa así en la esfera de la didáctica de la lengua, que promueve el encuentro de dos perspectivas: el diseño y la evaluación de propuestas de intervención, y el análisis de la interacción verbal (Cambra, 2003). Se parte de la idea según la cual en esta interacción se podrán apreciar las trazas observables de los procesos cognitivos de aprendizaje metalingüístico, asumiendo que el conocimiento no se puede referir nunca a una frase o a una estructura aislada, sino que depende de las acciones e interpretaciones de los participantes en el curso de la conversación (Coll y Solé, 1990; Barnes y Todd, 1995). Se pretende capturar el conocimiento generado en la interacción y explorar la relación dinámica que esta interacción tiene con el trabajo colaborativo y con las ayudas proporcionadas por el profesor (como por ejemplo: organización de las tareas, delimitación de objetivos, pautas y guías, materiales de autoayuda, etc., Piggot y Barr, 2002).

\section{Idea final}

En este texto hemos presentado un conjunto de reflexiones sobre la enseñanza de la gramática en el aula de lengua primera. A pesar de los problemas acertadamente señalados por un gran número de estudios, hemos visto de qué manera puede abordarse la gramática en la escuela obligatoria. Hemos intentado mostrar la importancia de atender los procesos de enseñanza y aprendizaje, así como los contenidos gramaticales. La integración de estos ámbitos permite ser optimista por cuanto abre nuevas avenidas de investigación y debate.

\section{Referencias Bibliográficas}

Andrews, R., Torgerson, C., Beverton, S., Freeman, A., Locke, T., Low, G., Robinson, A., y Zhu, D. (2004a). The effect of grammar teaching (syntax) in English on 5 to 16 year olds' accuracy and quality in written composition. London: EPPI-Centre, Social Science Research Unit, Institute of Education. [http://eppi.ioe.ac.uk/eppiwebcontent/reel/review groups/english/eng rv6/eng_rv6.pdf; consulta: diciembre de 2011]

Andrews, R., Torgerson, C., Beverton, S., Freeman, A., Locke, T., Low, G., Robinson, A., y Zhu, D. (2004b). The effect of grammar teaching (sentence combining) in English on 5 to 16 year olds' accuracy and quality in written composition: Review summary. University of York, UK. 
[http://www.york.ac.uk/media/educationalstudies/documents/research/EnglishGrammar \%28SC\%29.pdf; consulta: diciembre de 2011]

Andrews, R. (2010). Teaching sentence-level grammar for writing: The evidence so far. En T. Locke (Coord.) Beyond the grammar wars. A resource for teachers and students on developing language knowledge in the English/Literacy classroom (pp. 91-108). New York (NY): Routledge.

Barnes, D., y Todd, F. (1995). Communication and learning revisited. Making meaning through talk. Portsmouth: Boynton-Cook Publishers.

Bergin, D. (1999). Influences on classroom interest, Educational psychologist, 34(2): 87-98.

Bronckart, J. P. (2008). La actividad verbal, las alenguas y la lengua; reflexiones teóricas y didácticas. En A. Camps M. Milian (Coords.) Miradas y voces. Investigación sobre la educación lingüística y literaria en entornos plurilingües (pp. 27-44). Barcelona : Graó.

Bronckart, J. P., y Schneuwly, B. (1991). La didactique de la langue maternelle. L'émergence d'une utopie indispensable. Éducation et Recherche, 13: 8-26.

Brucart, J. M. (2000). L'anàlisi sintàctica i la seva terminologia en l'ensenyament secundari. En J. Macià y J. Solà (Coords.) La terminologia lingüística en l'ensenyament secundari. Propostes pràctiques (pp. 163-229). Barcelona : Graó.

Cambra, M. (2003). Une approche ethnographique de la classe de langue. Paris: Didier.

Cameron, D. (1995). Verbal hygiene. London: Routledge.

Camps, A. (1986). La gramàtica a l'escola bàsica, entre els 5 i els 10 anys. Barcelona: Barcanova.

Camps, A. (1994). L'ensenyament de la composició escrita. Barcelona: Barcanova.

Camps, A. (1998). L'ensenyament de la gramática. En A. Camps y T. Colomer (Coords.) L'ensenyament $i$ l'aprenentatge de la llengua i la literatura en l'educació secundària (pàgs. 105-126). Barcelona: ICE-UB / Horsori.

Camps, A. (2000). El coneixement gramatical dels alumnes: el cas dels pronoms personals. En J. Macià y J. Solà (Coords.) La terminologia lingüística en l'ensenyament secundari. Propostes practiques (pp. 121-136). Barcelona: Graó.

Camps, A. (2010a). Intervenció, innovació i investigación. Una relació necessària per a les didàctiques. En J. Vallès, D. Álvarez, R. Rickenmann (Coords.) L'activitat docent. Intervenció, innovació i investigación (pp. 115-124). Girona: Documenta universitaria.

Camps, A. (2010b). Hablar y reflexionar sobre la lengua: hacia un modelo de enseñanza de la gramática basado en la actividad reflexiva en colaboración. En T. Ribas (Coord.) Libros de texto y enseñanza de la gramática (pp. 13-31). Barcelona: Graó.

Camps, A., y Ferrer, M. (Coords.) (2000). Gramàtica a l'aula. Barcelona. Graó.

Camps, A., y Zayas, F. (Coords.) (2006). Secuencias didácticas para aprender gramática. Barcelona: Graó.

Camps, A., Guasch, O., Milian, M., y Ribas, T. (2005). Bases per a l'ensenyament de la gramàtica. Barcelona: Graó.

Coll, C., y Solé, I. (1990). La interacción profesor/alumno en el proceso de enseñanza y aprendizaje. En C. Coll, J. Palacios y A. Marchesi (Coords.), Desarrollo psicológico y educación. Vol. III (pp. 315-333). Madrid: Alianza.

Desclot, M. (1977). Una teoria sintàctica per a l'escola. Barcelona: Casals.

Dubois, J. (1969). Grammaire structurale du français: la phrase et les transformations. Paris: Loarousse.

Edwards, D., y N. Mercer (1987). Common knowledge: The development of understanding in the classroom. London: Routledge.

Fisher, C. (1996). Les savoirs grammaticaux des élèves du primaire: le cas de l'adjectif. En S.G. Chartrand (Coord.) Pour un nouvel enseignement de la grammaire (pp. 315-340). Québec: Les Éditions Logiques.

Fisher, C. (2004). La place des représentations des apprenants en didactique de la grammaire. En C. Vargas (Coord.) Langue et études de la langue. Approches linguistiques et didactiques (pp. 383-393). Aix-en-Provence : Publications de l'Université de Provence.

Fontich, X. (2006). Hablar y escribir para aprender gramática. Barcelona: ICE-Horsori. 
Fontich, X. (2010). La construcció del saber metalingüístic: estudi sobre l'aprenentatge de la gramàtica d'escolars de secundària en el marc d'una seqüència didàctica. Bellaterra. UAB [www.tdx.cat; consulta: diciembre de 2011]

Fontich, X. (2011). The construction of grammar knowledge. CORELA - RJC Cotexte, contexte, situation | Numéros thématiques. Consulta: 18 julio 2013.

Fontich, X. (2013, en publicación). Le verbe, centre de la phrase: Les étudiants classent des verbes en prenant compte des compléments verbaux. En C. Gomila et D. Ulma (Coords.) Le verbe en toute complexité: acquisition, transversalité et apprentissage. Paris: L'Harmattan.

Freeman, D., y Freeman, Y. (1998). Preparing teachers to teach about language. En C. Weaver (Coord.) Lessons to share (pp. 279-292). Portsmouth (NH): Heinemann.

French, R. (2010). Primary school children learning grammar: Rethinking the possibilities. En T. Locke (Coord.) Beyond the grammar wars. A resource for teachers and students on developing language knowledge in the English/Literacy classroom (pp. 206-229). New York (NY): Routledge.

Gil, R. (2010). Reflexionar sobre la llengua per aprendre a escriure. Un repte difícil però necessari, Guix, 371: 25-33.

Gil, R. (2011). Escriure textos reals en col-laboració: una tasca imprescindible, Guix, 363: 1016.

Gil, R., y Bigas, M. (2009). L'activitat metalingüística durant una tasca de segmentació d'un text en paraules a segon de primària. Articles de didàctica de la llengua i la literatura, 48: 2033.

Gonzalvo, L., y Camps, A. (2003). Els conceptes metalingüístics dels alumnes de secundària: el subjecte. Articles de didàctica de la llengua i la literatura, 31: 111-123.

Guasch, O. (2001). L'escriptura en segones llengües. Barcelona: Graó.

Janks, H. (2010). Language as a system of meaning potential: The reading and design of verbal texts. En T. Locke (Coord.) Beyond the grammar wars. A resource for teachers and students on developing language knowledge in the English/Literacy classroom (pp. 151169). New York (NY): Routledge.

Kilcher-Hagedorn, H., Othenin-Girard, C., y de Weck, G. (1987). Le savoir grammatical des élèves. Berne: Peter Lang.

Kumpulainen, K., y Mutanen, M. (2000). Mapping the dynamics of peer group interaction: A method analysis of social shared learning processes. En H. Cowie y G. van der Aalsvoort (Eds.) Social interaction in earning and instruction. The meaning of discourse for the construction of knowledge (pp. 141-161). Oxford: Pergamon-Earli.

Larsen-Freeman, D. (2003). Teaching language: from grammar to grammaring. Boston (MA): Heinle y Heinle.

Locke, T. (2009). Grammar and writing. The international debate. En R. Beard, D. Myhill, J. Riley y M. Nystrand (Coords.) The SAGE handbook of writing development (pp. 182193). London: SAGE Publications.

Locke, T. (Coord.) (2010a). Beyond the grammar wars. A resource for teachers and students on developing language knowledge in the English/Literacy classroom. New York (NY): Routledge.

Locke, T. (2010b). Discovering a metalanguage for all seasons: Bringing literary language in from the cold. En T. Locke (Coord.) Beyond the grammar wars. A resource for teachers and students on developing language knowledge in the English/Literacy classroom (pp. 170-184). New York (NY): Routledge.

Manresa, M., y Durán, C. (2011). Secuencias didácticas de lengua y literatura. En U. Ruiz Bikandi (Coord.) Lengua castellana y literatura. Investigación, innovación y buenas prácticas (pp. 195-213). Barcelona: Graó.

Martin, D. (1999). La terminologie grammaticale à l'école: facilitateur ou obstacle aux apprentissages? L'exemple de la suite du verbe. En M. J. Béguelin, J. F. de Pietro, y A. Näf, (Coords.) La terminologie grammaticale à l'école: perspectives interlinguistiques (pp. 13-35). Université de Neuchâtel : Institute de linguistique. 
Martínez Laínez, A. (2010) El tratamiento de la estructura del predicado en una muestra de libros de texto. En T. Ribas (Coord.) Libros de texto y enseñanza de la gramática (pp. 135-154). Barcelona: Graó.

Méndez, A. (2011). La reflexió gramatical sobre l'oració en una aula de primària. Bellaterra. GREAL [trabajo inédito].

Mercer, N. (2008). Talk and the development of reasoning and understanding. Human development, 51 (1): 90-100.

Mercer N., y Littleton, K. (2007). Dialogue and the development of children's thinking. A sociocultural approach. New York: Routledge.

Milian, M. (2004). Contextual factors enhancing cognitive and metacognitive activity during the process of collaborative writing. En G. Rijlarsdaam, H. van den Bergh y M. Couzijn (Coords.) Effective learning and teaching of writing. A handbook of writing in education (pp. 59-76). New York (NY): Kluwer Academic Publishers.

Milian, M. (2005). Parlar per fer gramàtica, Articles de didàctica de la llengua i la literatura, 37: 11-30.

Milian, M., y Camps, A. (2006). El razonamiento metalingüístico en el marco de secuencias didácticas de gramática (SDG). En A. Camps (Coord.) Diálogo e investigación en las aulas: investigaciones en didáctica de la lengua (pp. 25-54). Barcelona: Graó.

Myhill, D. (2000). Misconceptions and difficulties in the acquisition of metalinguistic knowledge, Language and education, 14(3): 151-163.

Myhill, D. (2011). Harnessing grammar: Weaving words and shaping Texts. Better: EvidenceBased Education. Winter: 12-13.

[http://betterevidence.files.wordpress.com/2011/03/better_language_arts_sample_article.p df, consulta: diciembre de 2011]

Nadeau, M., y Fisher, C. (2011). Les connaissances implicites et explicites en grammaire: quelle importance pour l'enseignement? Quelles conséquences? Bellaterra Journal of Teaching \& Learning Language \& Literature, 4(4): 1-31. [http://ojs.uab.cat/index.php/jtl3/article/view/446, consulta: diciembre 2011)

Notario, G. (2001). Los conceptos gramaticales de los alumnos de secundaria: el sujeto. En A. Camps (Coord.) El aula como espacio de investigación y reflexión (pp. 181-193). Barcelona: Graó.

Piggot, T. D., y Barr, R. (2002). Designing programmatic interventions. En M. L. Kamil, P. D. Pearson y R. Barr (Coords.) Handbook of reading research (vol. 3) (pp. 99-108). Mahwah (NJ): Lawrence Erlbaum.

Rabatel, A. (Coord.) (2004). Interactions orales en contexte didactique. Lyon : IUFM-Presses Universitaires de Lyon.

Ribas, T., Milian, M., Guasch, O., y Camps, A. (2002). La composición escrita como objeto de reflexión. En J.M. Cots y L. Nussbaum (Coords.) Pensar lo dicho. La reflexión sobre la lengua y la comunicación en el aprendizaje de lenguas (pp. 167-184). Lleida: Milenio.

Rodríguez, C. (2000). Els problemas de la transposición didáctica. La gramática des de dins de l'aula. En A. Camps (Coord.) Gramàtica a l'aula. Barcelona: Graó.

Rodríguez, C. (2011). El saber gramatical sobre los tiempos del pasado en alumnos de $4^{o}$ de Secundaria Obligatoria. Las relaciones entre conceptualización y uso reflexivo en la enseñanza de la gramática. València. Universitat de València [tesis doctoral no publicada].

Schwarz, B., Dreyfus, T., y Hershkowitz, R. (Coords.) (2009). Transformation of knowledge through classroom interaction. London y New York: Routledge.

Sinclair, J., y Coulthard, M. (1975). Towards an analysis of discourse. Oxford: Oxford University Press.

Trévise, A. (2010). Apostar per la inevitable activitat metalingüística dels estudiants en l'ensenyament de les llengües. En O. Guasch (Coord.) El tractament integrat de les àrees de llengua (pp. 131-144). Barcelona: Graó.

van Gelderen, A. (2010). Does explicit teaching of grammar help students to become better writers? Insights from empirical research. En T. Locke (Coord.) Beyond the 
grammar wars. A resource for teachers and students on developing language knowledge in the English/Literacy classroom (pp. 109-128). New York (NY): Routledge.

van Lier, L. (1995). Introducing 'language awareness'. London: Penguin.

van Lier, L. (2004). The ecology and semiotics of language learning. Dordrecht: Kluwer Academic Publishers.

van Lier, L. (2008). The ecology of language learning and sociocultural theory. In A. Creese, P. Martin y N. Hornberger (Coords.) Encyclopedia of language education. Vol. 9. Ecology of language (pp. 53-67). New York (NY): Springer.

Vargas, C. (2009). Peut-on inventer une grammaire pour la réussite scolaire? Mises en perspectives et voies de renouveau, Recherche \& Formation: La construction des savoirs grammaticaux, 39: 17-39.

Vilà, M. (Coord.) (2004). Didàctica de la llengua oral formal. Barcelona: Graó.

Weaver, C. (2010). Scaffolding grammar instruction for writers and writing. En T. Locke (Coord.) Beyond the grammar wars. A resource for teachers and students on developing language knowledge in the English/Literacy classroom (pp. 185-207). New York (NY): Routledge.

Wegerif, R., Boero, P., Andriessen, J., y Forman, E. (2009). A dialogue on dialogue and its place within education. En B. Schwartz, T. Dreyfus, y R. Hershkowitz (Coords.), Transformation of knowledge through classroom interaction (pp. 184-199). London, New York: Routledge.

Wells, G. (2001). Indagación dialógica. Barcelona: Paidós.

Zayas, F. (2006). Hacia una gramática pedagógica. En A. Camps y F. Zayas (Coords.) Secuencias didácticas para aprender gramática (pp. 17-30). Barcelona: Graó.

\footnotetext{
${ }^{1}$ Este artículo es una revisión y ampliación con permiso del editor del siguiente texto: Fontich, X. (2011). La enseñanza de la gramática en primaria y secundaria: algunas reflexiones y propuestas. $D a$ Investigação às Práticas, 1(2), 39 - 58.

${ }^{2}$ M. Milian, comunicación personal.

${ }^{3}$ Grup de Recerca sobre Ensenyament i Aprenentatge de Llengües (http://www.greal.cat/)
}

\begin{abstract}
Referencia del autor
Xavier Fontich Vicens es profesor de secundaria en un centro escolar en Barcelona y en el Departament de Didàctica de la Llengua i la Literatura, i de les Ciències Socials de la Universitat Autònoma de Barcelona. Ha publicado varios artículos y capítulos sobre la enseñanza de la literatura, la composición escrita, las nuevas tecnologías y la formación del profesorado. Su línea de trabajo principal es la enseñanza de la gramática en secundaria. Su tesis de doctorado (2010) aborda la importancia de la interacción oral en el aula en pequeño grupo para aprender gramática. Es autor de la obra Hablar y escribir para aprender gramática (Barcelona, Horsori, 2006) y en este momento participa como coeditor en un volumen que muestra los resultados de investigaciones actuales sobre la enseñanza de la gramática en España.

Email: xavier.fontich@uab.cat
\end{abstract}

Para citar este artículo:

Fontich Vicens, X. (2013). La gramática de la primera lengua en la escuela: Reflexiones sobre su enseñanza y su aprendizaje y sobre el contenido gramatical escolar. Bellaterra Journal of Teaching \& Learning Language \& Literature, 6(3), 1-19. 